\title{
SEVERAL CLOSED AND DETERMINANTAL FORMS FOR CONVOLVED FIBONACCI NUMBERS
}

\author{
MUHAMMET CIHAT DAĞLI AND FENG QI
}

\begin{abstract}
In the paper, with the aid of the Faà di Bruno formula, some properties of the Bell polynomials of the second kind, and a general derivative formula for a ratio of two differentiable functions, the authors establish several closed and determinantal forms for convolved Fibonacci numbers.
\end{abstract}

\section{CONTENTS}

1. Background and motivations 1

2. Lemmas 2

3. Main results 4

References 8

\section{BACKGROUND AND MOTIVATIONS}

The Fibonacci numbers $F_{n}$ are defined by the recurrence relation $F_{n}=F_{n-1}+$ $F_{n-2}$ for $n \geq 2$ subject to initial values $F_{0}=0$ and $F_{1}=1$. All the Fibonacci numbers $F_{n}$ for $n \geq 1$ can be generated by

$$
\frac{1}{1-t-t^{2}}=\sum_{n=0}^{\infty} F_{n+1} t^{n}=1+t+2 t^{2}+3 t^{3}+5 t^{4}+8 t^{5}+\cdots
$$

Numerous types of generalizations of these numbers have been offered in the literature and they have a plenty of interesting properties and applications to almost every field of science and art. For more information, please refer to [11, 18, 32] and closely related references therein.

For $r \in \mathbb{R}$, convolved Fibonacci numbers $F_{n}(r)$ can be defined by

$$
\frac{1}{\left(1-t-t^{2}\right)^{r}}=\sum_{n=0}^{\infty} F_{n+1}(r) t^{n}
$$

See [1, 2, 6, 14, 19] and closely related references therein. It is easy to see that $F_{n}(1)=F_{n}$ for $n \geq 1$. Convolved Fibonacci numbers $F_{n}(r)$ for $n \in \mathbb{N}$ and their generalizations have been considered in the papers [8, 10, 12, 14, 19, 37, 38, 39]

2010 Mathematics Subject Classification. 05A19, 11B83, 11C20, 11Y55.

Key words and phrases. Bell polynomial of the second kind; closed form; convolved Fibonacci number; determinantal form; Faà di Bruno formula; Fibonacci number; Hessenberg determinant; tridiagonal determinant.

This paper was typeset using $\mathcal{A M}_{\mathcal{M}} \mathcal{S}$-LATEX 
in a variety of context. For instance, Ramirez defined in [37 the convolved $h(x)$ Fibonacci numbers as an extension of classical convolved Fibonacci numbers and offered several combinatorial formulas.

In recent years, the second author of this paper and his colleagues have obtained a number of explicit and recursive formulas for some important numbers and polynomials via the Faà di Bruno formula, some properties of the Bell polynomials of the second kind, and a general derivative formula for a ratio of two differentiable functions. One may consult the papers [15, 16, 17, 21, 24, 28, 29, 30, 36, especially the review and survey article 31, and closely related references therein.

A tridiagonal determinant is a determinant whose nonzero elements locate only on the diagonal and slots horizontally or vertically adjacent the diagonal. In other words, a determinant $H=\left|h_{i j}\right|_{n \times n}$ is called a tridiagonal determinant if $h_{i j}=0$ for all pairs $(i, j)$ such that $|i-j|>1$. See [7].

A determinant $H=\left|h_{i j}\right|_{n \times n}$ is called a lower (or an upper, respectively) Hessenberg determinant if $h_{i j}=0$ for all pairs $(i, j)$ such that $i+1<j$ (or $j+1<i$, respectively). See [13].

In analytic combinatorics and analytic number theory, explaining a sequence of numbers or a sequence of polynomials in terms of a special and simple determinant is an interesting and important direction and sometimes produces significant results. Accordingly, some sequences and polynomials, such as the Bernoulli numbers and polynomials [22, 26], the Euler numbers and polynomials [40, the Delannoy numbers [15, 20, 21, 23, the Horadam polynomials [27, and the Fibonacci numbers and polynomial $[18,20,25,33$, have been considered in this framework. Interested readers can also refer to the studies in $[9,25,34,35$, and closely related references therein.

In mathematics, a closed form is a mathematical expression that can be evaluated in a finite number of operations and may contain constants, variables, four arithmetic operations, and elementary functions, but usually no limit. For systematic explanation of closed forms, please refer to the article [3] and closely related references therein.

In this paper, we will find several closed and determinantal forms for convolved Fibonacci numbers $F_{n+1}(r)$ by means of the Faà di Bruno formula, some properties and special values for the Bell polynomials of the second kind, and the generating function method, while we will give a determinantal formula for convolved Fibonacci numbers $F_{n+1}(r)$ in terms of tridiagonal determinants by aid of a general derivative formula, which is fundamental but not extensively circulated yet, for the ratio of two differentiable functions.

\section{LEMMAS}

In order to prove our main results, we recall several lemmas below.

Lemma 1 ([5, pp. 134 and 139]). The Bell polynomials of the second kind, or say, partial Bell polynomials, denoted by $\mathrm{B}_{n, k}\left(x_{1}, x_{2}, \ldots, x_{n-k+1}\right)$ for $n \geq k \geq 0$, is defined by

$$
\mathrm{B}_{n, k}\left(x_{1}, x_{2}, \ldots, x_{n-k+1}\right)=\sum_{\substack{1 \leq i \leq n \\ \ell_{i} \in\{0\} \cup \mathbb{N} \\ \sum_{i=1}^{n} i \ell_{i}=n \\ \sum_{i=1}^{n} \ell_{i}=k}}^{\infty} \frac{n !}{\prod_{i=1}^{\ell-k+1} \ell_{i} !} \prod_{i=1}^{\ell-k+1}\left(\frac{x_{i}}{i !}\right)^{\ell_{i}} .
$$


The Faà di Bruno formula can be described in terms of the Bell polynomials of the second kind $\mathrm{B}_{n, k}\left(x_{1}, x_{2}, \ldots, x_{n-k+1}\right)$ by

$$
\frac{\mathrm{d}^{n}}{\mathrm{~d} t^{n}} f \circ h(t)=\sum_{k=0}^{n} f^{(k)}(h(t)) \mathrm{B}_{n, k}\left(h^{\prime}(t), h^{\prime \prime}(t), \ldots, h^{(n-k+1)}(t)\right) .
$$

Lemma 2 ([5, p. 135]). For $n \geq k \geq 0$, we have

$$
\mathrm{B}_{n, k}\left(a b x_{1}, a b^{2} x_{2}, \ldots, a b^{n-k+1} x_{n-k+1}\right)=a^{k} b^{n} \mathrm{~B}_{n, k}\left(x_{1}, x_{2}, \ldots, x_{n-k+1}\right),
$$

where $a$ and $b$ are any complex number.

Lemma 3 ([31, Section 1.4]). For $n \geq k \geq 0$, we have

$$
\mathrm{B}_{n, k}(x, 1,0, \ldots, 0)=\frac{(n-k) !}{2^{n-k}}\left(\begin{array}{l}
n \\
k
\end{array}\right)\left(\begin{array}{c}
k \\
n-k
\end{array}\right) x^{2 k-n} .
$$

Lemma 4 ([22, Lemma 2.4]). Let $f(t)=1+\sum_{k=1}^{\infty} a_{k} t^{k}$ and $g(t)=1+\sum_{k=1}^{\infty} b_{k} t^{k}$ be formal power series such that $f(t) g(t)=1$. Then

$$
b_{n}=(-1)^{n}\left|\begin{array}{ccccccc}
a_{1} & 1 & 0 & \cdots & 0 & 0 & 0 \\
a_{2} & a_{1} & 1 & \cdots & 0 & 0 & 0 \\
a_{3} & a_{2} & a_{1} & \cdots & 0 & 0 & 0 \\
\vdots & \vdots & \vdots & \ddots & \vdots & \vdots & \vdots \\
a_{n-1} & a_{n-2} & a_{n-3} & \cdots & a_{1} & 1 & 0 \\
a_{n-1} & a_{n-2} & a_{n-3} & \cdots & a_{2} & a_{1} & 1 \\
a_{n} & a_{n-1} & a_{n-2} & \cdots & a_{3} & a_{2} & a_{1}
\end{array}\right| .
$$

Lemma 5 (4, p. 40, Entry 5]). For two differentiable functions $p(x)$ and $q(x) \neq 0$, we have

$$
\frac{\mathrm{d}^{k}}{\mathrm{~d} x^{k}}\left[\frac{p(x)}{q(x)}\right]=\frac{(-1)^{k}}{q^{k+1}}\left|\begin{array}{cccccc}
p & q & 0 & \cdots & 0 & 0 \\
p^{\prime} & q^{\prime} & q & \cdots & 0 & 0 \\
p^{\prime \prime} & q^{\prime \prime} & \left(\begin{array}{c}
2 \\
1
\end{array}\right) q^{\prime} & \cdots & 0 & 0 \\
\vdots & \vdots & \vdots & \ddots & 0 & 0 \\
p^{(k-2)} & q^{(k-2)} & \left(\begin{array}{c}
k-2 \\
1
\end{array}\right) q^{(k-3)} & \cdots & q & 0 \\
p^{(k-1)} & q^{(k-1)} & \left(\begin{array}{c}
1-1 \\
1
\end{array}\right) q^{(k-2)} & \cdots & \left(\begin{array}{c}
k-1 \\
k-2
\end{array}\right) q^{\prime} & q \\
p^{(k)} & q^{(k)} & \left(\begin{array}{c}
k \\
1
\end{array}\right) q^{(k-1)} & \cdots & \left(\begin{array}{c}
k \\
k-2
\end{array}\right) q^{\prime \prime} & \left(\begin{array}{c}
k \\
k-1
\end{array}\right) q^{\prime}
\end{array}\right| .
$$

In other words,

$$
\frac{\mathrm{d}^{k}}{\mathrm{~d} x^{k}}\left[\frac{p(x)}{q(x)}\right]=\frac{(-1)^{k}}{q^{k+1}(x)}\left|U_{(k+1) \times 1}(x) \quad V_{(k+1) \times k}(x)\right|,
$$

where the matrix $U_{(k+1) \times 1}(x)=\left(u_{\ell, 1}(x)\right)$ has the elements $u_{\ell, 1}(x)=p^{(\ell-1)}(x)$ for $1 \leq \ell \leq k+1$ and the matrix $V_{(k+1) \times k}(x)$ has the entries of the form

$$
v_{i j}(x)= \begin{cases}\left(\begin{array}{l}
i-1 \\
j-1
\end{array}\right) q^{(i-j)}(x), & i-j \geq 0 \\
0, & i-j<0\end{cases}
$$

for $1 \leq i \leq k+1$ and $1 \leq j \leq k$. 


\section{MAIN RESUlts}

In this section, we state and prove our main results.

Theorem 1. For $k \geq 0$ and $r \in \mathbb{R}$, convolved Fibonacci numbers $F_{k+1}(r)$ can be computed by

$$
F_{k+1}(r)=\sum_{\ell=0}^{k}\left(\begin{array}{c}
\ell \\
k-\ell
\end{array}\right) \frac{(r)_{\ell}}{\ell !}
$$

where

$$
(x)_{n}=\prod_{k=0}^{n-1}(x+k)= \begin{cases}x(x+1) \cdots(x+n-1), & n \geq 1 \\ 1, & n=0\end{cases}
$$

denotes the rising factorial, or say, Pochhammer symbol, of $x \in \mathbb{C}$. Consequently, the Fibonacci numbers $F_{k+1}$ for $k \geq 0$ can be computed by

$$
F_{k+1}=\sum_{\ell=0}^{k}\left(\begin{array}{c}
\ell \\
k-\ell
\end{array}\right)
$$

Proof. By virtue of (2), (3), and (4), we have

$$
\begin{aligned}
\frac{\mathrm{d}^{k}}{\mathrm{~d} t^{k}}\left[\frac{1}{\left(1-t-t^{2}\right)^{r}}\right] & =\sum_{\ell=0}^{k}\langle-r\rangle_{\ell}\left(1-t-t^{2}\right)^{-r-\ell} \mathrm{B}_{k, \ell}(-1-2 t,-2,0, \ldots, 0) \\
& =\sum_{\ell=0}^{k}\langle-r\rangle_{\ell}\left(1-t-t^{2}\right)^{-r-\ell}(-2)^{\ell} \mathrm{B}_{k, \ell}\left(t+\frac{1}{2}, 1,0, \ldots, 0\right) \\
& \rightarrow \sum_{\ell=0}^{k}\langle-r\rangle_{\ell}(-2)^{\ell} \mathrm{B}_{k, \ell}\left(\frac{1}{2}, 1,0, \ldots, 0\right), \quad t \rightarrow 0 \\
& =\sum_{\ell=0}^{k}\langle-r\rangle_{\ell}(-2)^{\ell} \frac{(k-\ell) !}{2^{k-\ell}}\left(\begin{array}{c}
k \\
\ell
\end{array}\right)\left(\begin{array}{c}
\ell \\
k-\ell
\end{array}\right)\left(\frac{1}{2}\right)^{2 \ell-k} \\
& =k ! \sum_{\ell=0}^{k}\langle-r\rangle_{\ell}(-1)^{\ell} \frac{1}{\ell !}\left(\begin{array}{c}
\ell \\
k-\ell
\end{array}\right) \\
& =k ! \sum_{\ell=0}^{k}(r)_{\ell} \frac{1}{\ell !}\left(\begin{array}{c}
\ell \\
k-\ell
\end{array}\right),
\end{aligned}
$$

where

$$
\langle x\rangle_{n}=\prod_{k=0}^{n-1}(x-k)= \begin{cases}x(x-1) \cdots(x-n+1), & n \geq 1 \\ 1, & n=0\end{cases}
$$

denotes the falling factorial of $x \in \mathbb{C}$. So, it follows from (1) that

$$
F_{k+1}(r)=\frac{1}{k !} \lim _{t \rightarrow 0} \frac{\mathrm{d}^{k}}{\mathrm{~d} t^{k}}\left[\frac{1}{\left(1-t-t^{2}\right)^{r}}\right]=\sum_{l=0}^{k} \frac{(r)_{\ell}}{\ell !}\left(\begin{array}{c}
\ell \\
k-\ell
\end{array}\right) .
$$

The proof of Theorem 1 is complete. 
Theorem 2 ([19, Theorem 1]). For $k \geq 0$ and $r \in \mathbb{R}$, convolved Fibonacci numbers $F_{k+1}(r)$ can be computed by

$$
F_{k+1}(r)=\frac{1}{k !} \frac{1}{\left(\frac{\sqrt{5}-1}{2}\right)^{k}} \sum_{\ell=0}^{k}(-1)^{\ell}\left(\begin{array}{l}
k \\
\ell
\end{array}\right)\left(\frac{\sqrt{5}-1}{\sqrt{5}+1}\right)^{\ell}(r)_{\ell}(r)_{k-\ell} .
$$

Consequently, the Fibonacci numbers $F_{k+1}$ for $k \geq 0$ can be computed by

$$
F_{k+1}=\frac{1}{\left(\frac{\sqrt{5}-1}{2}\right)^{k}} \sum_{\ell=0}^{k}(-1)^{\ell}\left(\frac{\sqrt{5}-1}{\sqrt{5}+1}\right)^{\ell} .
$$

Proof. It is easy to see that

$$
\frac{1}{1-t-t^{2}}=\frac{1}{\left(t+\frac{\sqrt{5}+1}{2}\right)\left(\frac{\sqrt{5}-1}{2}-t\right)}
$$

It is not difficult to see that

$$
\begin{aligned}
\frac{\mathrm{d}^{k}}{\mathrm{~d} t^{k}}\left[\frac{1}{(t+a)^{r}(b-t)^{r}}\right] & =\sum_{\ell=0}^{k}\left(\begin{array}{l}
k \\
\ell
\end{array}\right)\left[\frac{1}{(t+a)^{r}}\right]^{(\ell)}\left[\frac{1}{(b-t)^{r}}\right]^{(k-\ell)} \\
& =\sum_{\ell=0}^{k}\left(\begin{array}{l}
k \\
\ell
\end{array}\right) \frac{\langle-r\rangle_{\ell}}{(t+a)^{r+\ell}} \frac{\langle-r\rangle_{k-\ell}(-1)^{k-\ell}}{(b-t)^{r+k-\ell}} \\
& =\sum_{\ell=0}^{k}(-1)^{\ell}\left(\begin{array}{l}
k \\
\ell
\end{array}\right) \frac{(r)_{\ell}}{(t+a)^{r+\ell}} \frac{(r)_{k-\ell}}{(b-t)^{r+k-\ell}} \\
& \rightarrow \sum_{\ell=0}^{k}(-1)^{\ell}\left(\begin{array}{l}
k \\
\ell
\end{array}\right) \frac{(r)_{\ell}}{a^{r+\ell}} \frac{(r)_{k-\ell}}{b^{r+k-\ell}}, \quad t \rightarrow 0 .
\end{aligned}
$$

Accordingly, considering the first equality in (5), taking $a=\frac{\sqrt{5}+1}{2}$ and $b=\frac{\sqrt{5}-1}{2}$, and simplifying yield the formula (6). The proof of Theorem 2 is complete.

Theorem 3. For $k \geq 0$ and $r \in \mathbb{R}$, convolved Fibonacci numbers $F_{k+1}(r)$ can be computed by

$$
F_{k+1}(r)=(-1)^{k}\left|\begin{array}{ccccccc}
a_{1} & 1 & 0 & \cdots & 0 & 0 & 0 \\
a_{2} & a_{1} & 1 & \cdots & 0 & 0 & 0 \\
a_{3} & a_{2} & a_{1} & \cdots & 0 & 0 & 0 \\
\vdots & \vdots & \vdots & \ddots & \vdots & \vdots & \vdots \\
a_{k-1} & a_{k-2} & a_{k-3} & \cdots & a_{1} & 1 & 0 \\
a_{k-1} & a_{k-2} & a_{k-3} & \cdots & a_{2} & a_{1} & 1 \\
a_{k} & a_{k-1} & a_{k-2} & \cdots & a_{3} & a_{2} & a_{1}
\end{array}\right|
$$


and satisfy the identity

$$
\begin{aligned}
\sum_{\ell=0}^{k}(-1)^{\ell} \frac{\langle r\rangle_{\ell}}{\ell !}\left(\begin{array}{c}
\ell \\
k-\ell
\end{array}\right) & =(-1)^{k}\left|\begin{array}{ccccccc}
F_{1}(r) & 1 & 0 & \cdots & 0 & 0 & 0 \\
F_{2}(r) & F_{1}(r) & 1 & \cdots & 0 & 0 & 0 \\
F_{3}(r) & F_{2}(r) & F_{1}(r) & \cdots & 0 & 0 & 0 \\
\vdots & \vdots & \vdots & \ddots & \vdots & \vdots & \vdots \\
F_{k-2}(r) & F_{k-3}(r) & F_{k-4}(r) & \cdots & F_{1}(r) & 1 & 0 \\
F_{k-1}(r) & F_{k-2}(r) & F_{k-3}(r) & \cdots & F_{2}(r) & F_{1}(r) & 1 \\
F_{k}(r) & F_{k-1}(r) & F_{k-2}(r) & \cdots & F_{3}(r) & F_{2}(r) & F_{1}(r)
\end{array}\right| .
\end{aligned}
$$

where

$$
a_{k}=\sum_{\ell=0}^{k}(-1)^{\ell} \frac{\langle r\rangle_{\ell}}{\ell !}\left(\begin{array}{c}
\ell \\
k-\ell
\end{array}\right) .
$$

Proof. For $\left|t^{2}+t\right|<1$, using the binomial theorem leads to

$$
\begin{aligned}
\left(1-t-t^{2}\right)^{r} & =\sum_{k=0}^{\infty}\langle r\rangle_{k} \frac{(-1)^{k}}{k !}\left(t^{2}+t\right)^{k} \\
& =\sum_{k=0}^{\infty}\langle r\rangle_{k} \frac{(-1)^{k}}{k !} \sum_{\ell=0}^{k}\left(\begin{array}{l}
k \\
\ell
\end{array}\right) t^{k+\ell} \\
& =\sum_{k=0}^{\infty} \sum_{m=k}^{2 k}\langle r\rangle_{k} \frac{(-1)^{k}}{k !}\left(\begin{array}{c}
k \\
m-k
\end{array}\right) t^{m} \\
& =\sum_{m=0}^{\infty} \sum_{k=0}^{m}\langle r\rangle_{k} \frac{(-1)^{k}}{k !}\left(\begin{array}{c}
k \\
m-k
\end{array}\right) t^{m} \\
& =\sum_{k=0}^{\infty} \sum_{\ell=0}^{k}\langle r\rangle_{\ell} \frac{(-1)^{\ell}}{\ell !}\left(\begin{array}{c}
\ell \\
k-\ell
\end{array}\right) t^{k} .
\end{aligned}
$$

From the equation (1), it follows that

$$
\begin{aligned}
1 & =\left(1-t-t^{2}\right)^{r} \sum_{k=0}^{\infty} F_{k+1}(r) t^{k} \\
& =\left(\sum_{k=0}^{\infty}\left[\sum_{\ell=0}^{k}\langle r\rangle_{\ell} \frac{(-1)^{\ell}}{\ell !}\left(\begin{array}{c}
\ell \\
k-\ell
\end{array}\right)\right] t^{k}\right) \sum_{k=0}^{\infty} F_{k+1}(r) t^{k} .
\end{aligned}
$$

Further regarding coefficients in the above bracket as $a_{k}$ and $F_{k+1}(r)$ as $b_{k}$ in Lemma 4, interchanging the roles of $a_{k}$ and $b_{k}$, and simplifying arrive at desired expressions. 
Theorem 4. For $k \geq 0$ and $r \in \mathbb{R}$, convolved Fibonacci numbers $F_{k+1}(r)$ can be computed by

$$
F_{k+1}(r)=(-1)^{k}\left|\begin{array}{ccccccc}
1 & \gamma_{0} & 0 & 0 & \cdots & 0 & 0 \\
0 & \gamma_{1} & \gamma_{0} & 0 & \cdots & 0 & 0 \\
0 & \gamma_{2} & \left(\begin{array}{l}
2 \\
1
\end{array}\right) \gamma_{1} & \gamma_{0} & \cdots & 0 & 0 \\
0 & \gamma_{3} & \left(\begin{array}{l}
3 \\
1
\end{array}\right) \gamma_{2} & \left(\begin{array}{l}
3 \\
2
\end{array}\right) \gamma_{1} & \cdots & 0 & 0 \\
\vdots & \vdots & \vdots & \vdots & \ddots & \vdots & \vdots \\
0 & \gamma_{k-2} & \left(\begin{array}{c}
k-2 \\
1
\end{array}\right) \gamma_{k-3} & \left(\begin{array}{c}
k-2 \\
2
\end{array}\right) \gamma_{k-4} & \cdots & \gamma_{0} & 0 \\
0 & \gamma_{k-1} & \left(\begin{array}{c}
k-1 \\
1
\end{array}\right) \gamma_{k-2} & \left(\begin{array}{c}
k-1 \\
2
\end{array}\right) \gamma_{k-3} & \cdots & \left(\begin{array}{c}
k-1 \\
k-2
\end{array}\right) \gamma_{1} & \gamma_{0} \\
0 & \gamma_{k} & \left(\begin{array}{c}
k \\
1
\end{array}\right) \gamma_{k-1} & \left(\begin{array}{c}
k \\
2
\end{array}\right) \gamma_{k-2} & \cdots & \left(\begin{array}{c}
k \\
k-2
\end{array}\right) \gamma_{2} & \left(\begin{array}{c}
k \\
k-1
\end{array}\right) \gamma_{1}
\end{array}\right|,
$$

where

$$
\gamma_{k}=\gamma_{k}(r)=\sum_{\ell=0}^{k} \frac{\langle r\rangle_{\ell}}{\ell !}\left(\begin{array}{c}
\ell \\
k-\ell
\end{array}\right) .
$$

Proof. Proceeding as in the proof of Theorem 1 reveals

$$
\lim _{t \rightarrow 0} \frac{\mathrm{d}^{k}}{\mathrm{~d} t^{k}}\left[\left(1-t-t^{2}\right)^{r}\right]=k ! \sum_{\ell=0}^{k} \frac{\langle r\rangle_{\ell}}{\ell !}\left(\begin{array}{c}
\ell \\
k-\ell
\end{array}\right)=k ! \gamma_{k}(r) .
$$

By aid of Lemma 5 for $p(t)=1$ and $q(t)=\left(1-t-t^{2}\right)^{r}$, we have

$$
\begin{aligned}
& \frac{\mathrm{d}^{k}}{\mathrm{~d} t^{k}}\left[\frac{1}{\left(1-t-t^{2}\right)^{r}}\right]=\frac{(-1)^{k}}{\left(1-t-t^{2}\right)^{r(k+1)}} \\
& \times\left|\begin{array}{cccccc}
1 & q(t) & 0 & \cdots & 0 & 0 \\
0 & q^{\prime}(t) & q(t) & \cdots & 0 & 0 \\
0 & q^{\prime \prime}(t) & \left(\begin{array}{l}
2 \\
1
\end{array}\right) q^{\prime}(t) & \cdots & 0 & 0 \\
\vdots & \vdots & \vdots & \ddots & \vdots & \vdots \\
0 & q^{(k-2)}(t) & \left(\begin{array}{c}
k-2 \\
1
\end{array}\right) q^{(k-3)}(t) & \ldots & q(t) & 0 \\
0 & q^{(k-1)}(t) & \left(\begin{array}{c}
k-1 \\
1
\end{array}\right) q^{(k-2)}(t) & \cdots & \left(\begin{array}{c}
k-1 \\
k-2
\end{array}\right) q^{\prime}(t) & q(t) \\
0 & q^{(k)}(t) & \left(\begin{array}{c}
k \\
1
\end{array}\right) q^{(k-1)}(t) & \cdots & \left(\begin{array}{c}
k \\
k-2
\end{array}\right) q^{\prime \prime}(t) & \left(\begin{array}{c}
k \\
k-1
\end{array}\right) q^{\prime}(t)
\end{array}\right| \\
& \rightarrow(-1)^{k}\left|\begin{array}{cccccc}
1 & \gamma_{0} & 0 & \ldots & 0 & 0 \\
0 & \gamma_{1} & \gamma_{0} & \ldots & 0 & 0 \\
0 & \gamma_{2} & \left(\begin{array}{l}
2 \\
1
\end{array}\right) \gamma_{1} & \ldots & 0 & 0 \\
\vdots & \vdots & \vdots & \ddots & \vdots & \vdots \\
0 & \gamma_{k-2} & \left(\begin{array}{c}
k-2 \\
1
\end{array}\right) \gamma_{k-3} & \ldots & \gamma_{0} & 0 \\
0 & \gamma_{k-1} & \left(\begin{array}{c}
k-1 \\
1
\end{array}\right) \gamma_{k-2} & \ldots & \left(\begin{array}{c}
k-1 \\
k-2
\end{array}\right) \gamma_{1} & \gamma_{0} \\
0 & \gamma_{k} & \left(\begin{array}{c}
k \\
1
\end{array}\right) \gamma_{k-1} & \ldots & \left(\begin{array}{c}
k \\
k-2
\end{array}\right) \gamma_{2} & \left(\begin{array}{c}
k-1 \\
k-1
\end{array}\right) \gamma_{1}
\end{array}\right|
\end{aligned}
$$

as $t \rightarrow 0$. Using the first equality in (5), we find that

$$
F_{k+1}(r)=\frac{1}{k !} \lim _{t \rightarrow 0} \frac{\mathrm{d}^{k}}{\mathrm{~d} t^{k}}\left[\frac{1}{\left(1-t-t^{2}\right)^{r}}\right]
$$




$$
=(-1)^{k}\left|\begin{array}{cccccc}
1 & \gamma_{0} & 0 & \ldots & 0 & 0 \\
0 & \gamma_{1} & \gamma_{0} & \ldots & 0 & 0 \\
0 & \gamma_{2} & \left(\begin{array}{l}
2 \\
1
\end{array}\right) \gamma_{1} & \ldots & 0 & 0 \\
\vdots & \vdots & \vdots & \ddots & \vdots & \vdots \\
0 & \gamma_{k-2} & \left(\begin{array}{c}
k-2 \\
k
\end{array}\right) \gamma_{k-3} & \ldots & \gamma_{0} & 0 \\
0 & \gamma_{k-1} & \left(\begin{array}{c}
k-1 \\
1
\end{array}\right) \gamma_{k-2} & \ldots & \left(\begin{array}{c}
k-1 \\
k-2
\end{array}\right) \gamma_{1} & \gamma_{0} \\
0 & \gamma_{k} & \left(\begin{array}{c}
k \\
1
\end{array}\right) \gamma_{k-1} & \ldots & \left(\begin{array}{c}
k \\
k-2
\end{array}\right) \gamma_{2} & \left(\begin{array}{c}
k \\
k-1
\end{array}\right) \gamma_{1}
\end{array}\right| .
$$

The proof of Theorem 4 is complete.

\section{REFERENCES}

[1] G. E. Bergum and V. E. Jr. Hoggatt, Limits of quotients for the convolved Fibonacci sequence and related sequences, Fibonacci Quart. 15 (1977), 113-116.

[2] G. E. Bergum and V. E. Jr. Hoggatt, Numerator polynomial coefficient array for the convolved Fibonacci sequence, Fibonacci Quart. 14 (1976), 43-48.

[3] J. M. Borwein and R. E. Crandall, Closed forms: what they are and why we care, Notices Amer. Math. Soc. 60 (2013), no. 1, 50-65; available online at https://doi.org/10.1090/ noti936.

[4] N. Bourbaki, Functions of a Real Variable, Elementary Theory, Translated from the 1976 French original by Philip Spain. Elements of Mathematics (Berlin). Springer-Verlag, Berlin, 2004; available online at https://doi.org/10.1007/978-3-642-59315-4

[5] L. Comtet, Advanced Combinatorics: The Art of Finite and Infinite Expansions, Revised and Enlarged Edition, D. Reidel Publishing Co., 1974; available online at https://doi.org/ 10.1007/978-94-010-2196-8

[6] H. W. Corley, The convolved Fibonacci equation, Fibonacci Quart. 27 (1989), 283-284.

[7] V. Higgins and C. Johnson, Inverse spectral problems for collections of leading principal submatrices of tridiagonal matrices, Linear Algebra Appl. 489 (2016), 104-122; available online at https://doi.org/10.1016/j.laa.2015.10.004

[8] V. E. Jr. Hoggatt and M. Bicknell-Johnson, Fibonacci convolution sequences, Fibonacci Quart. 15 (1977), no. 2, 117-122.

[9] A. İpek and K. Arı, On Hessenberg and pentadiagonal determinants related with Fibonacci and Fibonacci-like numbers, Appl. Math. Comput. 229 (2014), 433-439; available online at https://doi.org/10.1016/j.amc.2013.12.071.

[10] T. Kim, D. V. Dolgy, D. S. Kim, and J. J. Seo, Convolved Fibonacci numbers and their applications, Ars Combin. 135 (2017), 119-131.

[11] T. Koshy, Fibonacci and Lucas Numbers with Applications, Pure and Applied Mathematics (New York), Wiley-Interscience, New York, 2001; available online at https://doi.org/10. 1002/9781118033067

[12] G. Liu, Formulas for convolution Fibonacci numbers and polynomials, Fibonacci Quart. 40 (2002), no. 4, 352-357.

[13] R. S. Martin and J. H. Wilkinson, Handbook Series Linear Algebra: Similarity reduction of a general matrix to Hessenberg form, Numer. Math. 12 (1968), no. 5, 349-368; available online at https://doi.org/10.1007/BF02161358.

[14] P. Moree, Convoluted convolved Fibonacci numbers, J. Integer Seq. 7 (2004), Article 04.2.2, 14 pages.

[15] F. Qi, A determinantal expression and a recursive relation of the Delannoy numbers, Acta Univ. Sapientiae Math. 12 (2020), no. 2, in press; arXiv prprint (2020), available online at https://arxiv.org/abs/2003.12572

[16] F. Qi, An explicit formula for the Bell numbers in terms of the Lah and Stirling numbers, Mediterr. J. Math. 13 (2016), no. 5, 2795-2800; available online at https://doi.org/10. 1007/s00009-015-0655-7

[17] F. Qi, Derivatives of tangent function and tangent numbers, Appl. Math. Comput. 268 (2015), 844-858; available online at http://dx.doi.org/10.1016/j.amc.2015.06.123

[18] F. Qi, Denying a short proof of a determinantal formula for generalized Fibonacci polynomials, J. Math. Anal. 11 (2020), no. 1, 52-57. 
[19] F. Qi, Three closed forms for convolved Fibonacci numbers, Eur. J. Pure Appl. Math. (2020), in press; OSF Preprints (2020), available online at https://doi.org/10.31219/osf .io/9gqrb

[20] F. Qi, V. Čerňanová, and Y. S. Semenov, Some tridiagonal determinants related to central Delannoy numbers, the Chebyshev polynomials, and the Fibonacci polynomials, Politehn. Univ. Bucharest Sci. Bull. Ser. A Appl. Math. Phys. 81 (2019), no. 1, 123-136.

[21] F. Qi, V. Čerňanová, X.-T. Shi, and B.-N. Guo, Some properties of central Delannoy numbers, J. Comput. Appl. Math. 328 (2018), 101-115; available online at https://doi.org/10.1016/ j.cam.2017.07.013.

[22] F. Qi and R. J. Chapman, Two closed forms for the Bernoulli polynomials, J. Number Theory 159 (2016), 89-100; available online at https://doi.org/10.1016/j.jnt.2015.07.021

[23] F. Qi, M. C. Dağll, and W.-S. Du, Determinantal forms and recursive relations of the Delannoy two-functional sequence, OSF Preprints (2020), available online at https://doi.org/ 10.31219/osf.io/u683y

[24] F. Qi and B.-N. Guo, Explicit formulas for special values of the Bell polynomials of the second kind and for the Euler numbers and polynomials, Mediterr. J. Math. 14 (2017), no. 3, Article 140, 14 pages; available online at https://doi.org/10.1007/s00009-017-0939-1

[25] F. Qi and B.-N. Guo, Expressing the generalized Fibonacci polynomials in terms of a tridiagonal determinant, Matematiche (Catania) 72 (2017), no. 1, 167-175; available online at https://doi.org/10.4418/2017.72.1.13

[26] F. Qi and B.-N. Guo, Some determinantal expressions and recurrence relations of the Bernoulli polynomials, Mathematics 4 (2016), no. 4, Article 65, 11 pages; available online at https://doi.org/10.3390/math4040065

[27] F. Qi, C. Kizllates,, and W.-S. Du, A closed formula for the Horadam polynomials in terms of a tridiagonal determinant, Symmetry 11 (2019), no. 6, 8 pages; available online at https: //doi.org/10.3390/sym11060782

[28] F. Qi, O. Kouba, and I. Kaddoura, Computation of several Hessenberg determinants, Math. Slovaca 70 (2020), in press; OSF Preprints (2020), available online at https://doi.org/10. 31219/osf.io/x4unj

[29] F. Qi, D. Lim, and B.-N. Guo, Explicit formulas and identities for the Bell polynomials and a sequence of polynomials applied to differential equations, Rev. R. Acad. Cienc. Exactas Fís. Nat. Ser. A Mat. RACSAM 113 (2019), no. 1, 1-9; available online at https://doi.org/10. 1007/s13398-017-0427-2

[30] F. Qi, D.-W. Niu, and B.-N. Guo, Some identities for a sequence of unnamed polynomials connected with the Bell polynomials, Rev. R. Acad. Cienc. Exactas Fís. Nat. Ser. A Math. RACSAM 113 (2019), no. 2, 557-567; available online at https://doi.org/10.1007/ s13398-018-0494-z

[31] F. Qi, D.-W. Niu, D. Lim, and Y.-H. Yao, Special values of the Bell polynomials of the second kind for some sequences and functions, J. Math. Anal. Appl. ??? (2020), no. ?, in press; available online at https://www.researchgate.net/publication/342751624

[32] F. Qi, E. Polatli, and B.-N. Guo, Determinantal formulas and recurrent relations for biperiodic Fibonacci and Lucas polynomials, OSF Preprints (2020), available online at https: //doi.org/10.31219/osf.io/qvxd8

[33] F. Qi, J.-L. Wang, and B.-N. Guo, A determinantal expression for the Fibonacci polynomials in terms of a tridiagonal determinant, Bull. Iranian Math. Soc. 45 (2019), no. 6, 1821-1829; available online at https://doi.org/10.1007/s41980-019-00232-4

[34] F. Qi, J.-L. Wang, and B.-N. Guo, A representation for derangement numbers in terms of a tridiagonal determinant, Kragujevac J. Math. 42 (2018), no. 1, 7-14; available online at https://doi.org/10.5937/KgJMath1801007F

[35] F. Qi, J.-L. Zhao, and B.-N. Guo, Closed forms for derangement numbers in terms of the Hessenberg determinants, Rev. R. Acad. Cienc. Exactas Fís. Nat. Ser. A Mat. RACSAM 112 (2018), no. 4, 933-944; available online at https://doi.org/10.1007/s13398-017-0401-z

[36] F. Qi and M.-M. Zheng, Explicit expressions for a family of the Bell polynomials and applications, Appl. Math. Comput. 258 (2015), 597-607; available online at https://doi.org/ 10.1016/j.amc.2015.02.027

[37] J. L. Ramírez, On convolved generalized Fibonacci and Lucas polynomials, Appl. Math. Comput. 229 (2014), 208-213; available online at https://doi.org/10.1016/j.amc.2013.12.049 
[38] J. L. Ramírez, Some properties of convolved $k$-Fibonacci numbers, International Scholarly Research Notices 2013, Article ID 759641, 5 pages; available online at http://dx.doi.org/ 10.1155/2013/759641

[39] A. Şahin and J. L. Ramírez, Determinantal and permanental representations of convolved Lucas polynomials, Appl. Math. Comput. 281 (2016), 314-322; available online at https: //doi.org/10.1016/j.amc.2016.01.064

[40] C.-F. Wei and F. Qi, Several closed expressions for the Euler numbers, J. Inequal. Appl. 2015, Paper No. 219, 8 pages; available online at https://doi.org/10.1186/s13660-015-0738-9

Department of Mathematics, Akdeniz University, 07058-Antalya, Turkey

Email address: mcihatdagli@akdeniz.edu.tr

$U R L$ : https://orcid.org/0000-0003-2859-902X

Institute of Mathematics, Henan Polytechnic University, Jiaozuo 454010, Henan, China; College of Mathematics and Physics, Inner Mongolia University for Nationalities, Tongliao 028043, Inner Mongolia, China; School of Mathematical Sciences, Tianjin Polytechnic University, Tianjin 300387, China

Email address: qifeng618@gmail.com, qifeng618@hotmail.com, qifeng618@qq.com

$U R L$ : https://qifeng618.wordpress.com, https://orcid.org/0000-0001-6239-2968 